\title{
Penicillin Resistant Bacteremic Pneumococcal Pneumonia
}

\author{
Hugh G. Robson*, M.D.
}

\section{THE CASE}

A 76 year old man presented to the emergency room of the Royal Victoria Hospital (Montreal, Quebec, Canada) on January 18, 1998 because of worsening dyspnea and nonproductive cough. He was known to have end-stage lung disease with interstitial pulmonary fibrosis of more than 15 years duration. For the three months prior to hospital admission, he required continuous home oxygen treatment; severe dyspnea was precipitated by only slight effort. His medication included metered doses of ipratropium bromide and salbutamol, furosemide and $20 \mathrm{mg}$ prednisone daily.

Prior to his retirement he had worked for many years in the construction industry and had been heavily exposed to cement and dust. There was no record of his having annual influenza vaccination nor of receipt of polyvalent pneumococcus vaccine.

Physical examination in the emergency room showed the following vital signs: oral temperature $36^{\circ} \mathrm{C}$, pulse $80 /$ minute with frequent ectopic beats, blood pressure $100 / 70 \mathrm{mmHg}$ and respiratory rate 30/minute. Clubbing of the fingers was not noted, the trachea was central, and he had a barrel chest. Loud crackles were present bilaterally over the mid to lower lung zones. No signs of heart failure were recorded and the remainder of the examination was noncontributory.

Initial laboratory testing gave the following results: hemoglobin $163 \mathrm{~g} / \mathrm{L}$, leukocytes $12.7 \times 10^{9} / \mathrm{L}$, neutrophils $9.98 \times 10^{9} / \mathrm{L}$. In mmol/L, serum creatinine was 119 , serum sodium 137, potassium 3.7, chloride 93 and bicarbonate 32.6. Arterial blood gases at $\mathrm{FiO}_{2} 21 \%$ were $\mathrm{pO}_{2} 57 \mathrm{mmHg}, \mathrm{pCO}_{2} 42 \mathrm{mmHg}$ and $\mathrm{pH}$ 7.47. On

\footnotetext{
* To whom correspondence should be addressed: Microbiologist-inChief, Division of Infectious Diseases, Royal Victoria Hospital, 687, avenue des Pins Ouest, Montreal, Quebec, Canada, H3A 1A1
}

$40 \%$ oxygen by mask, ear oximetry showed the oxygen saturation to be $92 \%$. A chest radiograph demonstrated extensive interstitial disease with honey combing, but no alveolar disease, air bronchograms or pleural fluid were noted. The patient was transferred to the medical ward within 24 hours where his therapy included $40 \%$ oxygen by mask, aerosolized salbutamol, predinisone and furosemide. On the third hospital day, the prednisone dosage was increased and maintained at 60 $\mathrm{mg}$ orally per day. Over the first nine days of hospital stay his condition remained unchanged with continued dyspnea on slight effort, he was rousable and alert but required continuous $40 \%$ oxygen by mask to maintain his oxygen saturation at $92-95 \%$.

About $0200 h$, January 28, his condition abruptly changed. He became agitated and confused, oral temperature rose to $38.5^{\circ} \mathrm{C}$, with respirations of 40/minute. Lung auscultation revealed no changes but a repeat chest radiograph demonstrated a new left lower lobe infiltrate with air bronchograms consistent with pneumonia. Two blood cultures were obtained and intravenous antibiotic therapy with ticarcillin/clavulanic acid was begun. He became unresponsive and died within 6 hours. Later that day the blood cultures were positive showing Gram-positive cocci in pairs and short chains. The organism was identified as Streptococcus pneumoniae, type 14 . Susceptibility testing showed the following minimal inhibitory concentrations (MIC): penicillin $2 \mathrm{mg} / \mathrm{L}$ (resistant), ceftriaxone $2 \mathrm{mg} / \mathrm{L}$ (resistant), erythromycin $0.06 \mathrm{mg} / \mathrm{L}$ (susceptible) and vancomycin $0.5 \mathrm{mg} / \mathrm{L}$ (susceptible).

Autopsy confirmed the presence of extensive bilateral bronchopneumonia and end-stage pulmonary fibrosis. In addition, adenocarcinoma of the lung with foci in both right and left lower lobes and in a right hilar lymph node was discovered. The heart was enlarged showing 
biventricular dilatation and moderate right ventricular hypertrophy.

\section{DISCUSSION}

Although many of the features of pneumococcal pneumonia were well known to Osler (1), the disease and causative agent continued to evolve and display new facets. Still common and widespread, it remains "friend of the aged" with death rates increasing markedly after age 50, particularly in cases with documented bacteremia. Osler described lobar pneumonia as "the most widespread and the most fatal of all acute diseases" (1). Pneumonia of all causes is still the sixth leading cause of death in the United States, and pneumococcal pneumonia remains an important contributor to pneumonia morbidity and mortality (2). The importance of underlying disease, in particular alcoholism, was known to Osler, but was given added emphasis in the classic review by Austrian and Gold (3). The patient described in the current report had numerous factors known to confer a poor prognosis: age over 65, chronic lung disease, immunosuppression, neoplastic disease, respiratory rate over 30 per minute, temperature over $38.3^{\circ} \mathrm{C}$ and $\mathrm{pO}_{2}$ less than $60 \mathrm{mmHg}$ while breathing room air. The disease is often rapidly fatal in spite of appropriate antimicrobial therapy. Indeed such therapy may have little benefit for patients destined to die within the first few days of the illness (3). Austrian and Gold, who made this observation, were strong advocates for the reevaluation and widespread use of active immunization with polyvalent pneumococcal vaccine. As demonstrated by this patient, many people who are vaccine candidates due to predisposing factors have not received either influenza or pneumococcal vaccine. The patient's isolate was a type 14 strain, one of the capsular polysaccharide antigens included in the currently available pneumococcal vaccine.

The past decade has seen rapid emergence and spread of antibiotic resistance worldwide. As recently as 1990 penicillin resistance was infrequent in pneumococci in Montreal; most such isolates were characterized as penicillin-intermediate (4). In the past two years resistance has become increasingly common at the Royal Victoria Hospital approaching 20\% (H.G. Robson, unpublished observation). Many such isolates are now truly resistant with penicillin and cephalosporin MIC of $2 \mathrm{mg} / \mathrm{L}$ or above. At the currently observed levels of resistance it has not been shown that treatment of pneumonia with penicillin or cephalosporin antibiotics fails provided large antibiotic doses are used (5), but with further increases in resistance this remains a serious possibility.

\section{REFERENCES}

1. Osler W. Lobar pneumonia In: The Principles and Practice of Medicine. Third Edition, New York: D. Appleton and Company; 1898: 108-137.

2. Bartlett JG, Mundy LM. Community-acquired pneumonia. New England Journal of Medicine 333: 1618-1624; 1995.

3. Austrian R, Gold J. Pneumococcal bacteremia with especial reference to bacteremic pneumococcal pneumonia. Annals of Internal Medicine 60: 759-776; 1964.

4. Loo VG, Lavallée J, McAlear D, Robson HG. The in-vitro susceptibilities of 326 Streptococcus pneumoniae isolates to nine antimicrobial agents including penicillin and newer quinolones. Journal of Antimicrobial Chemotherapy 33: 641645; 1994.

5. Pallares R, Linares J, Vadillo M, et al. Resistance to penicillin and cephalosporin and mortality from severe pneumococcal pneumonia in Barcelona, Spain. New England Journal of Medicine. 333: 474-480; 1995.

Hugh G. Robson, M.D., graduated from the Faculty of Medicine, McGill University (Montreal, Quebec, Canada) with the class of 1960. He is currently the Microbiologist-in-Chief at the Royal Victoria Hospital (Montreal, Quebec, Canada). 